\title{
A new method to detect geometrical information by the tunneling microscope
}

\author{
Tasaki, S.; Levitan, J.; Mygind, Jesper
}

Published in:

Journal of Applied Physics

Link to article, DOI:

10.1063/1.366215

Publication date:

1997

Document Version

Publisher's PDF, also known as Version of record

Link back to DTU Orbit

Citation (APA):

Tasaki, S., Levitan, J., \& Mygind, J. (1997). A new method to detect geometrical information by the tunneling microscope. Journal of Applied Physics, 82(9), 4148-4152. https://doi.org/10.1063/1.366215

\section{General rights}

Copyright and moral rights for the publications made accessible in the public portal are retained by the authors and/or other copyright owners and it is a condition of accessing publications that users recognise and abide by the legal requirements associated with these rights.

- Users may download and print one copy of any publication from the public portal for the purpose of private study or research.

- You may not further distribute the material or use it for any profit-making activity or commercial gain

- You may freely distribute the URL identifying the publication in the public portal

If you believe that this document breaches copyright please contact us providing details, and we will remove access to the work immediately and investigate your claim. 


\title{
A new method to detect geometrical information by the tunneling microscope
}

\author{
Shuichi Tasaki \\ Kyoto University, Kyoto 606-01, Japan \\ Jacob Levitan ${ }^{\text {a) }}$ \\ Bar-Ilan University, Ramat-Gan 52900, Israel \\ Jesper Mygind \\ Technical University of Denmark, DK-2800, Lyngby, Denmark
}

(Received 4 November 1996; accepted for publication 28 July 1997)

\begin{abstract}
A new method for the detection of the geometrical information by the scanning tunneling microscope is proposed. In addition to the bias voltage, a small ac modulation is applied. The nonlinear dependence of the transmission coefficient on the applied voltage is used to generate harmonics. The ratio of the harmonics to the dc current is found to give the width between the sample and the probe, i.e., the geometrical information. This method may be useful to measure materials, where the local-spatial-density of states may change notably from place to place.

(C) 1997 American Institute of Physics. [S0021-8979(97)05621-1]
\end{abstract}

\section{INTRODUCTION}

Since the discovery of the scanning tunneling microscope (STM), ${ }^{1}$ it is widely used to investigate the surface structure of various materials (see e.g., Refs. 2-5). More recently, it is also used to manipulate individual atoms and molecules. ${ }^{6,7}$

Because of the exponential dependence of the tunneling current as a function of the tip-sample separation, the spatial resolution is extremely high. The tip can be used to track the surface topography as it is scanned, or conversely, the tip can be held fixed and track the motion of the surface. ${ }^{8}$ This is utilized in nanosize accelerometers with a feedback loop maintaining a constant tunneling current and tracking the motion of a cantilever beam. ${ }^{8-11}$ In the conventional method, the distance between the needle probe and substance is regulated so as to keep the tunneling current constant. ${ }^{9,10}$ However, the tunneling current depends not only on the distance between the probe and the substance but also on the electronic states of the substance. Therefore, the so-determined width profile contains both geometrical and electrical information.

There are indeed three factors which affect the tunneling current: The local density of states of the sample; the density of states of the tip; and the barrier penetration factor. ${ }^{12,13}$ Since the tunneling microscope displays the local density of electronic states it is crucial to obtain as exact information of those states as possible. This is complicated, however, due to the fact that these states are generally not simply related to the atom core position, and the states observed depend on the tip bias. Surface topography is best defined in terms of those core positions. Since no probe microscope directly measures core positions, the position of the surface must be calculated with theoretical models. This is at best a difficult task. ${ }^{14}$

\footnotetext{
a) Also at The Research Institute, The College of Judea and Samaria, 44837, Ariel, Israel.
}

In this article, we shall propose a new method based on the response of the tunneling current to the ac bias field. Because of the nonlinear dependence of the current on the bias voltage, higher harmonics are created. The amplitude of the second harmonic $j_{2 w}$ relative to the dc component of the current $j_{\mathrm{dc}}$ is shown to be

$$
\frac{j_{2 \omega}}{j_{\mathrm{dc}}}=\frac{w \nu_{d}}{4 d_{p}\left|E_{f}\right|}\left[\frac{\nu_{a}}{\nu_{d}}\right]^{2}
$$

where $\nu_{a}$ and $\nu_{d}$ are amplitudes of the ac and the dc parts of the applied voltage, $w$ is the distance from probe to material, $\left|E_{f}\right|$ is the work function, and $d_{p} \equiv\left\{2 m\left|E_{f}\right| / \hbar^{2}\right\}^{-1}$ the penetration depth of the wave function into the gap with $m$ being the electron mass. [Strictly speaking, Eq. (1.1) is valid for $\left(w \nu_{d}\right) /\left(d_{p}\left|E_{f}\right|\right) \ll 1$. For details see Sec. III.] The control parameter is the voltage across the gap, $\nu \equiv \epsilon w$, where $\epsilon$ is an applied (constant) electric field. Note that Eq. (1.1) does not contain the density of states for probe or sample. Therefore, we can directly obtain the geometrical information as expressed by $w$ by measuring the ratio between the second harmonic and the dc currents. The key point of picking up only the geometrical information is to measure the ratio of the currents instead of their absolute values.

In the following, we shall derive Eq. (1.1) by using a one-dimensional model. An extension to three-dimensional geometry is straightforward. We assume that both the applied ac voltage and the tunneling are very weak.

In Sec. II, our model will be explained and the transmission coefficient will be obtained in the Wentzel-KramersBrillouin (WKB) approximation. Then the transmission current will be calculated. In Sec. III, the response of the current under the voltage,

$$
\nu=\nu_{d}+\nu_{a} \cos (\omega t),
$$

will be studied and the new method of detecting the geometrical information will be explained. 


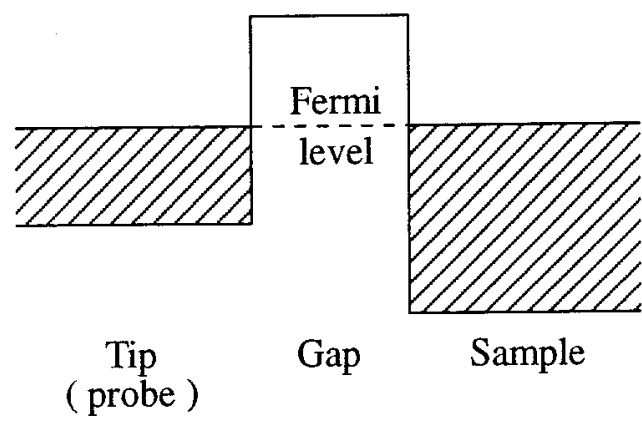

FIG. 1. Potential in equilibrium. The energy felt by an electron is indicated by the ordinate. The shaded regions stand for the occupied levels.

\section{TUNNELING CURRENT IN STM}

In the following we consider the case where the sample is a metal, and describe it by a one-dimensional model. We also assume that the effective electron masses of the sample and the metal tip are equal to the electron mass in vacuum.

In this section, we shall derive the tunneling current across the STM under a constant bias $E$ field. The derivation of the tunneling current between two metals separated by a thin insulating film has been performed by Simmons in Ref. 15. The following derivation is, however, more adequate for the present purpose. For a comparison of different approaches to the calculation of the tunneling through onedimensional potential barriers, see Ref. 16.

\section{A. Potential shape and model}

In the STM, the wave functions for the tip and for the sample can overlap in the gap region. The electron gases of the tip and the sample reach an equilibrium by inducing surface charges. This situation is shown in Fig. 1 and can be described by the Hamiltonian

$$
H=-\frac{\hbar^{2}}{2 m} \frac{d^{2}}{d x^{2}}+V(x)
$$

where the potential $V(x)$ is given by

$$
V(x)=\left\{\begin{array}{lr}
-V_{0}^{L}, \quad(x \leqslant 0) \\
0, \quad(0<x<w) \\
-V_{0}^{R}, \quad(x \geqslant w)
\end{array}\right.
$$

$V_{0}^{L}$ and $V_{0}^{R}$ being positive. The potential and the coordinate which we use are shown in Fig. 2.

When we apply a constant voltage (the sample side is positive), the potential near the gap will change, as shown in Fig. 3, and the potential becomes

$$
V(x)=\left\{\begin{array}{l}
-V_{0}^{L}, \quad(x \leqslant 0) \\
-e \epsilon x, \quad(0<x<w) . \\
-V_{0}^{R}-e \epsilon x . \quad(x \geqslant w)
\end{array} .\right.
$$

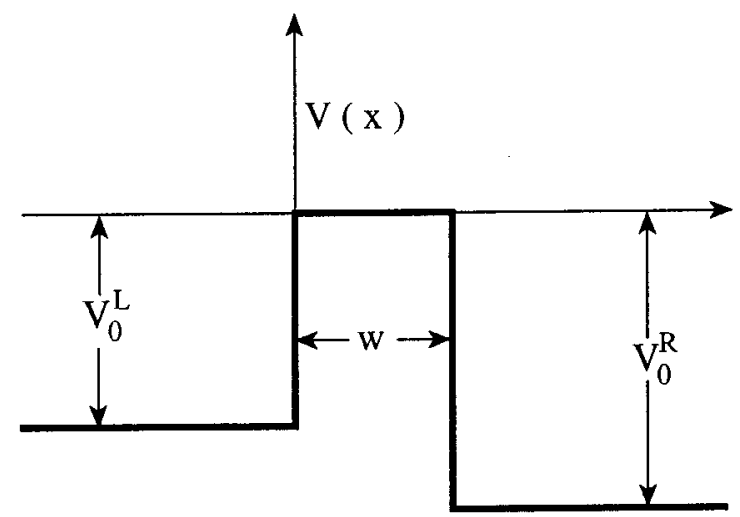

$$
\begin{aligned}
& \text { Tip Gap Sample } \\
& \text { (probe) }
\end{aligned}
$$

FIG. 2. Potential function $V(x)$ and the coordinate system used in the calculations. The energy felt by an electron is indicated by the ordinate.

Note that in the new situation, the energy differences between the band bottom and the Fermi level do not change as compared with the equilibrium case for both metal tip and sample.

The variation in $x$ is quite small. This is due to the smallness of $w$ combined with the weak slope of the trapezoidal potential. One can, without loss of generality, replace $V(x)$ by the average value in the interval $0 \leqslant x \leqslant w$. This leads to (an almost) constant current and voltage, and hence to a constant $\epsilon$.

\section{B. WKB calculation of the transmission coefficient}

We shall now calculate the transmission coefficient in the case of a constant bias field. We must therefore solve the Schroedinger equation:

$$
\frac{d^{2} \psi}{d x^{2}}+\frac{2 m}{\hbar^{2}}\{E-V(x) \psi\}=0
$$

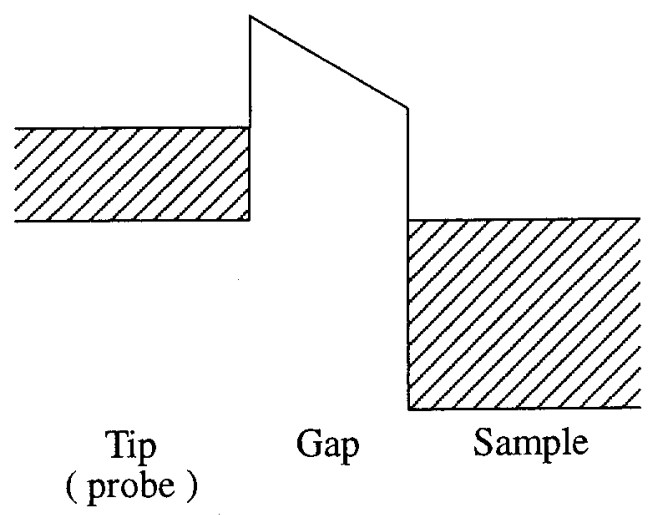

FIG. 3. Potential under the bias field (the sample side is positive). The energy felt by an electron is indicated by the ordinate. The shaded regions stand for the occupied levels. 
Since the change of the potential can be considered as slow, we shall solve (2.3) by the WKB method. In this method, the wave function $\psi(x)$ is expressed as

$$
\psi(x)= \begin{cases}\frac{A}{\sqrt{k(x)}} \exp \left\{i \int_{0}^{x} k(x) d x\right\}+\frac{B}{\sqrt{k(x)}} \exp \left\{-i \int_{0}^{x} k(x) d x\right\}, \quad(x \leqslant 0) \\ \frac{\mathrm{C}}{\sqrt{\kappa(\mathrm{x})}} \exp \left\{-\int_{0}^{x} \kappa(x) d x\right\}+\frac{D}{\sqrt{\kappa(x)}} \exp \left\{\int_{0}^{x} \kappa(x) d x\right\}, \quad(0<x<w) \\ \frac{\mathrm{F}}{\sqrt{\mathrm{k}(\mathrm{x})}} \exp \left\{i \int_{w}^{x} k(x) d x\right\}+\frac{G}{\sqrt{k(x)}} \exp \left\{-i \int_{w}^{x} k(x) d x\right\}, \quad(0 \leqslant w<x)\end{cases}
$$

where

$$
\begin{aligned}
& k(x)=\sqrt{\frac{2 m}{\hbar^{2}}\{E-\widetilde{V}(x)\}}, \\
& \kappa(x)=\sqrt{\frac{2 m}{\hbar^{2}}\{\widetilde{V}(x)-E\} .}
\end{aligned}
$$

By applying the connection formula to the left and to the right region in Eq. (2.4b), we get the transmission coefficient from left to right, setting $G=0$.

$$
\begin{aligned}
& T_{L \rightarrow R}(E)=T_{R \rightarrow L}(E)=\frac{4}{\left(2 \theta+\frac{1}{2 \theta}\right)^{2}} \equiv T(E), \\
& \theta=\exp \left\{\sqrt{\frac{8 m e \epsilon}{9 \hbar^{2}}}\left[\left(\frac{|E|}{e \epsilon}\right)^{3 / 2}-\left(\frac{|E|}{e \epsilon}-w\right)^{3 / 2}\right]\right\} .
\end{aligned}
$$

In the limit of $|E| \gg e \epsilon w, \theta$ can be approximated as

$$
\theta \cong \exp \left(\frac{2 m|E|}{\hbar^{2}} w\right) \exp \left(-\frac{1}{4} \sqrt{\frac{2 m|E|}{\hbar^{2}}} w \frac{e \epsilon w}{|E|}\right) .
$$

Note that, in (2.6), there exists no restriction about the magnitude of $\theta$, in contrast to the Bardeens formula (see discussion of Ref. 3). By using the path integral method, the present result can be extended to three-dimensional cases.

\section{Tunneling current}

The electrons in the left hand side with energy $E$ moving to the right can tunnel to the right hand side. The number of such electrons is given by

$$
2 \frac{\Omega}{h} d k_{L} f_{L}(E), \quad\left(k_{L}>0\right),
$$

where $\Omega$ is the volume of the left hand side, $f_{L}(E)$ the Fermi distribution in the left hand side, and the factor 2 comes from the spin degrees of freedom. The flux of such electrons is given by Eq. (2.7) by substituting $\nu_{L}=\hbar k_{L} / m$ into $\Omega$,

$$
d F_{L}=2 \frac{d k_{L}}{h} \frac{\hbar k_{L}}{m} f_{L}(E)=\frac{k_{L} d k_{L}}{\pi m} f_{L}(E),
$$

which gives the transmission current

$$
\begin{aligned}
j_{L \rightarrow R} & =-e \int_{k_{L}>0} d F_{L} T_{L \rightarrow R}\left[1-f_{R}(E)\right] \\
& =-e \int_{0}^{\infty} d k_{L} \frac{k_{L}}{\pi m} T_{L \rightarrow R} f_{L}(E)\left[1-f_{R}(E)\right],
\end{aligned}
$$

where $f_{R}(E)$ is the Fermi distribution function of the right hand side and is introduced in order to take the Pauli principle into account. Similarly, we have

$$
j_{R \rightarrow L}=+e \int_{0}^{\infty} d k_{R} \frac{k_{R}}{\pi m} T_{R \rightarrow L} f_{R}(E)\left[1-f_{L}(E)\right],
$$

and thus the total current $j$ across the junction is given by

$$
\begin{aligned}
j= & j_{L \rightarrow R}+j_{R \rightarrow L} \\
= & \frac{-e}{\pi \hbar^{2}}\left\{\int_{-V_{0}^{L}}^{\infty} d E T(E) f_{L}(E)\left[1-f_{R}(E)\right]\right. \\
& \left.-\int_{-V_{0}^{R}-e \epsilon w}^{\infty} d E T(E) f_{R}(E)\left[1-f_{L}(E)\right]\right\} \\
\cong & \frac{-e}{\pi \hbar^{2}} \int_{-\infty}^{\infty} d E T(E)\left[f_{L}(E)-f_{R}(E)\right],
\end{aligned}
$$

where we have assumed

$$
f_{L}(E)=f_{R}(E)=1 \quad \text { for } E<\max \left\{-V_{0}^{L},-V_{0}^{R}-e \epsilon w\right\} .
$$

The distribution functions $f_{L}(E)$ and $f_{R}(E)$ at temperature $T_{e}$ are given by

$$
\begin{aligned}
& f_{L}(E)=\left[\exp \left(\frac{E-E_{f}}{k_{B} T_{e}}\right)+1\right]^{-1}, \\
& f_{R}(E)=\left[\exp \left(\frac{E-E_{f}+e \epsilon w}{k_{B} T_{e}}\right)+1\right]^{-1},
\end{aligned}
$$

where $E_{f}$ is the Fermi energy measured from the vacuum and $k_{B}$ is the Boltzmann constant. Note that $\left|E_{f}\right|$ corresponds to the work function (see Fig. 1). Now let us assume $\left|E_{f}\right|$ $\gg e \epsilon w$. Then

$$
f_{L}(E)-f_{R}(E) \cong-e \epsilon w \frac{\partial f_{L}(E)}{\partial E},
$$


and the right hand side has a sharp peak at $E=E_{f}$. Thus we get

$$
j \cong-\frac{e^{2} \epsilon w}{\pi \hbar^{2}} T\left(E_{f}\right) .
$$

This is the desired result.

We finally get from Eq. (2.6)

$$
\begin{aligned}
j \cong & -\frac{e^{2} \epsilon w}{\pi \hbar^{2}} \exp \left(-2 \sqrt{\frac{2 m\left|E_{f}\right|}{\hbar^{2}}} w\right) \\
& \times \exp \left(\frac{1}{2} \sqrt{\frac{2 m\left|E_{f}\right|}{\hbar^{2}}} w \frac{e \epsilon w}{\left|E_{f}\right|}\right),
\end{aligned}
$$

which we shall use in the next section.

\section{RESPONSE UNDER THE AC FIELD}

It is convenient to rewrite the current $j$ in terms of the control parameter and the system variables. The control parameter is the voltage across the gap

$$
\nu \equiv \epsilon w,
$$

and the system variables are the penetration depth $d_{p}$ of electrons

$$
d_{p} \equiv\left(\frac{2 m\left|E_{f}\right|}{\hbar^{2}}\right)^{-1 / 2},
$$

the with $w$ of the gap and the work function $\left|E_{f}\right|$. In terms of those, we have

$$
j=-\frac{e}{\pi \hbar^{2}} \nu \exp \left(-2 \frac{w}{d_{p}}\right) \exp \left(\frac{w}{2 d_{p}} \frac{\nu}{\left|E_{f}\right|}\right) .
$$

Although formula (3.3) is derived for a static field, we can use it also for a low-frequency ac field by replacing the static voltage $\nu$ with the time-dependent one. Now we consider the current under the field

$$
\nu=\nu_{d}+\nu_{a} \cos (\omega t),
$$

where $\nu_{a} \ll \nu_{d}$. By substituting Eq. (3.4) into Eq. (3.3) and expanding it with respect to $\nu_{a}$, we obtain up to order $\left(\nu_{a} / \nu_{d}\right)^{2}$

$$
\begin{aligned}
j= & j_{d}\left[1+\frac{w}{4 d_{p}} \frac{\nu_{d}}{\left|E_{f}\right|}\left(1+\frac{w}{4 d_{p}} \frac{\nu_{d}}{\left|E_{f}\right|}\right)\left(\frac{\nu_{a}}{\nu_{d}}\right)^{2}\right] \\
& +j_{d}\left(1+\frac{w}{2 d_{p}} \frac{\nu_{d}}{\left|E_{f}\right|}\right) \frac{\nu_{a}}{\nu_{d}} \cos (\omega t)+j_{d} \frac{w}{4 d_{p}} \frac{\nu_{d}}{\left|E_{f}\right|} \\
& \times\left(1+\frac{w}{4 d_{p}} \frac{\nu_{d}}{\left|E_{f}\right|}\right)\left(\frac{\nu_{a}}{\nu_{d}}\right)^{2} \cos (2 \omega t),
\end{aligned}
$$

where

$$
j_{d}=-\frac{e}{\pi \hbar^{2}} \nu_{d} \exp \left(-2 \frac{w}{d_{p}}\right) \exp \left(\frac{w}{2 d_{p}} \frac{\nu_{d}}{\left|E_{f}\right|}\right) .
$$

Note that the current $j$ given by Eq. (3.5) contains harmonics due to the nonlinear dependence of the transmission coefficient on the external field. From Eq. (3.5), we obtain

$$
\begin{aligned}
& \frac{j_{w}}{j_{\mathrm{dc}}} \cong\left(1+\frac{w \nu_{d}}{2 d_{p}\left|E_{f}\right|}\right) \frac{\nu_{a}}{\nu_{d}}, \\
& \frac{j_{2 w}}{j_{\mathrm{dc}}} \cong \frac{w \nu_{d}}{4 d_{p}\left|E_{f}\right|}\left(1+\frac{w \nu_{d}}{4 d_{p}\left|E_{f}\right|}\right)\left(\frac{\nu_{a}}{\nu_{d}}\right)^{2},
\end{aligned}
$$

where $j_{\mathrm{dc}}, j_{w}$, and $j_{2 w}$ are the current amplitudes of the dc-component, the ac-component with frequency $\omega$, and the ac-component with frequency $2 \omega$, respectively.

Formula (3.7) can be used to obtain the value of $w /\left(d_{p}\left|E_{f}\right|\right)$ and thus of $w$ since $d_{p}^{-1}=\sqrt{2 m\left|E_{f}\right|} / \hbar$ is determined from the work function $\left|E_{f}\right|$ and the electron mass $m$ in the vacuum. In particular, in the case where $w \nu_{d}$ $\ll d_{p}\left|E_{f}\right|$, Eq. (3.7b) reduces to

$$
\frac{j_{2 w}}{j_{\mathrm{dc}}} \cong \frac{w \nu_{d}}{4 d_{p}\left|E_{f}\right|}\left(\frac{\nu_{a}}{\nu_{d}}\right)^{2}
$$

and directly gives the desired quantity.

The application of an ac dither on top of the dc bias voltage indeed is a standard technique used for $d I / d V$ measurements ${ }^{17}$ as well as noise suppression by phase sensitive detection (PSD). It is important to discuss the experimental conditions which are to be fulfilled in order to use the nonlinear characteristics of the tunneling $I V$ curve to generate the second harmonic. The frequency and the amplitude of the applied ac modulation should not in itself modulate the $I V$ curve, i.e., it should not alter the "distance" between the tip and the anvil. For this $f_{\text {ac }} \gg f_{\text {app }}$, where $f_{\text {app }}$ is the dominant cutoff frequency of the apparatus. The cutoff may be dictated by mechanical properties of the scanner tube or it may be the effective cutoff frequency imposed by the electrical feedback loop controlling the $z$ distance via the tunnel current whichever gives the highest cutoff. A modulation of the tip-anvil distance by the ac modulation will lead to parametric effects and may impose chaotic behavior, instabilities, and eventually excessive noise. The relatively high $f_{\text {ac }}$ may cause experimental problems with opening up the circuit for noise at higher frequencies. However, proper narrow band filtering of the tunnel current amplifier input leads should resolve this calamity. Except for the experimental problems mentioned above the measurement of dc and second harmonic is quite straightforward. The modulation frequency presumably is limited by the available phase sensitive detectors. Modern digitally based PSDs operate at several MHz.

An appealing alternative is to apply simultaneously two modulation frequencies. This allows for a double frequency detection scheme where the mixing product and eventually also the phase shift may give further information on the geometrical properties of the tunnel distance.

With two modulation frequencies the field has the form

$$
\nu=\nu_{d}+\nu_{a}\left(\alpha \cos \omega_{1} t+\beta \cos \omega_{2} t\right)
$$

and the current is given by 


$$
\begin{aligned}
j= & j_{d}\left[1+\frac{w}{4 d_{p}} \frac{\nu_{d}}{\left|E_{f}\right|}\left(1+\frac{w}{4 d_{p}} \frac{\nu_{d}}{\left|E_{f}\right|}\right)\left(\frac{\nu_{a}}{\nu_{d}}\right)^{2}\right]+j_{d}\left(1+\frac{w}{2 d_{p}} \frac{\nu_{d}}{\left|E_{f}\right|}\right) \frac{\nu_{a}}{\nu_{d}}\left[\cos \left(\omega_{1}, t\right) \alpha+\cos \left(\omega_{2} t\right) \beta\right] \\
& +j_{d} \frac{w}{4 d_{p}} \frac{\nu_{d}}{\left|E_{f}\right|}\left(1+\frac{w}{4 d_{p}} \frac{\nu_{d}}{\left|E_{f}\right|}\right)\left(\frac{\nu_{a}}{\nu_{d}}\right)^{2}\left\{\cos \left(2 \omega_{1} t\right) \alpha^{2}+\cos \left(2 \omega_{2} t\right) \beta^{2}+2 \alpha \beta\left[\cos \left(\omega_{1}+\omega_{2}\right) t+\cos \left(\omega_{1}-\omega_{2}\right) t\right]\right\},
\end{aligned}
$$

where

$$
j_{d}=-\frac{e}{\pi \hbar^{2}} \nu_{d} \exp \left(-2 \frac{w}{d_{p}}\right) \exp \left\{\frac{w}{2 d_{p}} \frac{\nu_{d}}{\left|E_{f}\right|}\right\} .
$$

Finally we get

$$
\frac{j_{\omega_{1}}(t)}{j_{\omega_{2}}(t)}=\frac{\omega \nu_{a}}{4 d_{p}\left|E_{f}\right|}\left[\frac{\alpha^{2} \cos 2 \omega_{1} t+\beta^{2} \cos 2 \omega_{2} t+2 \alpha \beta\left[\cos \left(\omega_{1}+\omega_{2}\right) t\right]+\cos \left(\omega_{1}-\omega_{2}\right) t}{\alpha \cos \omega_{1} t+\beta \cos \omega_{2} t}\right],
$$

with $\omega$ determined by Eq. (3.1).

One can also measure the current difference $j_{\omega_{1}-\omega_{2}}$ (or $\left.j_{\omega_{1}+\omega_{2}}\right)$ and determine the ratio, $j_{\omega_{1}-\omega_{2}} / j_{\omega_{1}}$. This is from an experimental point of view more feasible because of the lower frequency and the possibility still to remain "above" the $1 / f$ noise, but the expression one obtains is more complicated than Eq. (3.11).

\section{CONCLUSION}

The present method has some obvious advantages:

(1) Since we consider the ratio of the dc and the ac current, we can avoid the effect of the density of states which enters as a prefactor for the current.

(2) This method gives direct information on the transmission coefficient, for which the one-electron theory is expected to give an accurate prediction. This is due to the fact that the transmission beam is very sparse and that the electron-electron interactions can be neglected.

(3) In addition one has the possibility to apply simultaneously two modulation frequencies and hence obtain a double frequency detection scheme, where the mixing product may give further information on the geometrical properties of the tunneling distance.

Let us now compare the present method with the conventional one. In the conventional method, the width profile $w(\mathbf{r})$ is determined such that the tunneling current is kept constant. However, as already pointed out in Sec. I, the current also depends on the (local) density of states, and the so-determined width profile reflects not only the geometrical shape, but also the local electronic states. ${ }^{18}$ On the contrary, the present method just gives information about the geometrical shape, as we are not concerned with the absolute value of the current but the ratio between the $\mathrm{dc}$ and the ac components of the current. In this sense, the present method, which to the best of our knowledge has never previously been explored, is preferable to the conventional one. Moreover, by combining the two methods, we may determine both geometrical and electronic structures.

${ }^{1}$ G. Binnig and H. Rohrer, Helv. Phys. Acta 55, 726 (1982).

${ }^{2}$ A. A. Lucas, Europhys. News 21, 63 (1990).

${ }^{3}$ F. Besenbacher and K. Mortensen, Europhys. News 21, 68 (1990).

${ }^{4}$ R. Magno and M. G. Spencer, J. Appl. Phys. 72, 5333 (1992).

${ }^{5}$ S. K. Theiss, D. M. Chen, and J. A. Golovchenko, Appl. Phys. Lett. 66, 448 (1995).

${ }^{6}$ S. Heike, T. Hashizume, and Y. Wada, J. Appl. Phys. 80, 4182 (1996).

${ }^{7}$ Y. Wada, T. Uda, M. Lutwyche, S. Kondo, and S. Heike, J. Appl. Phys. 74, 7321 (1993).

${ }^{8}$ A. Morean and J. B. Ketterson, J. Appl. Phys. 72, 861 (1992).

${ }^{9}$ S. B. Waltman and W. J. Kaiser, Sens. Actuators 19, 201 (1989).

${ }^{10}$ S. B. Waltman and W. J. Kaiser, J. British Interplanetary Soc. 42, 474 (1989).

${ }^{11}$ T. E. Feuchtwang and P. H. Cutler, Phys. Scr. 35, 132 (1987).

${ }^{12}$ J. E. Griffith and D. A. Grigg, J. Appl. Phys. 74, 83 (1993).

${ }^{13}$ J. Tersoff and D. R. Hamann, Phys. Rev. B 31, 805 (1985).

${ }^{14}$ J. A. Stroscio, R. M. Feenstra, D. M. Newns, and A. P. Fein, J. Vac. Sci. Technol. A 6, 499 (1988).

${ }^{15}$ J. G. Simmons, J. Appl. Phys. 34, 1793 (1963).

${ }^{16}$ K. L. Jensen and A. K. Ganguly, J. Appl. Phys. 73, 4409 (1993).

${ }^{17}$ R. S. Becker, J. A. Golovchenko, and B. S. Swartzentruber, Phys. Rev. Lett. 55, 987 (1985).

${ }^{18}$ J. Tersoff, Phys. Rev. Lett. 57, 440 (1986). 